BULLETIN OF THE

AMERICAN MATHEMATICAL SOCIETY

Volume 79, Number 4, July 1973

\title{
ON A CONJECTURE OF M. KAC ${ }^{1}$
}

BY DANIEL W. STROOCK

Communicated by I. M. Singer, January 11,1973

Introduction. Consider the region $\mathscr{G}=\left\{(x, y, z) \in R^{3}: x^{2}+y^{2} \leqq f^{2}(z)\right.$, $0 \leqq z \leqq L\}$ obtained by rotating the curve $f(z), 0 \leqq x \leqq L$, about the $z$-axis. Denote by $q\left(t, \vec{r}, \vec{r}^{\prime}\right)$ the fundamental solution of the heat equation in $\mathscr{G}$ with zero boundary value on $\partial \mathscr{G}$, and let $p\left(t, \vec{r}, \vec{r}^{\prime}\right)=\left(1 /(2 \pi t)^{3 / 2}\right)$. $\exp \left(-\left|\vec{r}-\vec{r}^{\prime}\right|^{2} / 2 t\right)$. Express everything in terms of cylindrical coordinates $(\rho, \theta, z)$. The purpose of this note is to show that, for each $m \geqq 0$,

$$
\frac{\left.\int_{0}^{L} d z \int_{0}^{f(z)} \rho d \rho \frac{\partial^{2 m}}{\partial \theta^{2 m}} q(t,(\rho, 0, z),(\rho, \theta, z))\right|_{\theta=0}}{\left.\int_{0}^{L} d z \int_{0}^{f(z)} \rho d \rho \frac{\partial^{2 m}}{\partial \theta^{2 m}} p(t,(\rho, 0, z),(\rho, \theta, z))\right|_{\theta=0}} \rightarrow 1
$$

as $t>0$. As Kac points out in [2], this equation enables one to recapture the distribution of the function $f(z)$ from the eigenvalues of the Laplacian, with zero boundary conditions, in $\mathscr{G}$. Hence, one gets a refined version of Weyl's theorem (cf. Kac [1]) by taking advantage of the cylindrical symmetry of $\mathscr{G}$. Indeed, Weyl's theorem results from (1) with $m=0$. As will be seen, (1) can be viewed as an extended "principle of not feeling the boundary."'

The "principle of not feeling the boundary" is the statement that $q(t, \vec{r}, \vec{r}) / p(t, \vec{r}, \vec{r}) \rightarrow 1$ as $t>0$ for $\vec{r} \in \mathscr{G}$. Combining this with the maximum principle, which tells us that $q(t, \vec{r}, \vec{r}) \leqq p(t, \vec{r}, \vec{r})$, one can easily derive

$$
\int_{\mathscr{G}} q(t, \vec{r}, \vec{r}) d \ddot{r} / \int_{\mathscr{G}} p(t, \vec{r}, \vec{r}) d \vec{r} \rightarrow 1 .
$$

The derivation of (1) can be accomplished in the same way, only there is now an extra complication. The maximum principle can no longer be invoked to compare the $\theta$-derivatives of $q$ and $p$. For this reason we introduce a special representation of $q$ and $p$. From this representation it will be possible to conclude that

AMS (MOS) subject classifications (1970). Primary 35P15, 35J25; Secondary 60J65

${ }^{1}$ Results obtained at the Courant Institute of Mathematical Sciences, New York University, with the Air Force Office of Scientific Research Grant AF-AFOSR-71-2055.

Reproduction in whole or in part is permitted for any purpose of the United States Government. 
(2) $\left.\left|\frac{\partial^{2 m}}{\partial \theta^{2 m}} q(t,(\rho, 0, z),(\rho, \theta, z))\right|_{\theta=0}|\leqq| \frac{\partial^{2 m}}{\partial \theta^{2 m}} p(t,(\rho, 0, z),(\rho, \theta, z))\right|_{\theta=0} \mid+\frac{C_{m}}{t}$

for all $\rho$ and $z$. It will then be easy to get (1).

Basic representation. Let $\Omega=C([0, \infty),(0, \infty) \times R)$ and denote by $\rho(t, \omega)$ and $z(t, \omega)$ the first and second coordinates, respectively, of the path $\omega$ at time $t$. Set $\mathscr{M}_{t}=\mathscr{B}[(\rho(s), z(s)): s \leqq t]$ and $\mathscr{M}=\sigma\left(\bigcup_{t \geqq 0} \mathscr{M}_{t}\right)$. For each $(\rho, z) \in(0, \infty) \times R$ there is a unique probability measure $P_{\rho, z}$ on $\langle\Omega, \mathscr{M}\rangle$ such that $\beta_{1}(t)=\rho(t)-\rho-\int_{0}^{t}(1 / \rho(s)) d s$ and $\beta_{2}(t)=z(t)-z$ are independent $P_{\rho, z}$-Brownian motions (i.e., $P_{\rho, z}\left(\beta_{i}(0)=0\right)=1$ and $P_{\rho, z}\left(\beta_{i}(t+s) \in \Gamma \mid \mathscr{M}_{s}\right)=\int_{\Gamma} g\left(t, y-\beta_{i}(s)\right) d y, \quad i=1,2, \quad$ where $g(t, y)=$ $\left.(2 \pi t)^{-1 / 2} \exp \left(-y^{2} / 2 t\right)\right)$. H. P. McKean [3] is a good reference for such matters.

Next define

$$
\Theta(t, \theta)=\frac{1}{(2 \pi t)^{1 / 2}} \sum_{n=-\infty}^{\infty} \exp \left(-(\theta+2 \pi n)^{2} / 2 t\right) .
$$

Then $\Theta\left(t, \theta-\theta^{\prime}\right)$ is the transition probability density for Brownian motion on $S^{\prime}$. Let $R(t)=\int_{0}^{t}\left(1 / \rho^{2}(s)\right) d s$.

Using Itô's formula and Doob's stopping time theorem, one can easily derive

(3) $p\left(t,(\rho, \theta, z),\left(\rho^{\prime}, \theta^{\prime}, z^{\prime}\right)\right)=E_{\rho, z}\left[\Theta\left(R(t), \theta-\theta^{\prime}\right), \rho(t)=\rho^{\prime}, z(t)=z^{\prime}\right]$

and

(4) $q\left(t,(\rho, \theta, z),\left(\rho^{\prime}, \theta^{\prime}, z^{\prime}\right)\right)=E_{\rho, z}\left[\Theta\left(R(t), \theta-\theta^{\prime}\right), \rho(t)=\rho^{\prime}, z(t)=z^{\prime}, \tau>t\right]$,

where $\tau=\inf \{t \geqq 0: \rho(t) \geqq f(z(t))\}$. The expressions on the right-hand sides of (3) and (4) are meant to denote the densities of the corresponding quantities with respect to $\rho^{\prime} d \rho^{\prime} d z$.

Note that

$$
\begin{aligned}
& \frac{\partial^{2 m} \Theta}{\partial \theta^{2 m}}(t, \theta) \\
& \quad=(-1)^{m} \sum_{k=0}^{m}(-1)^{k} \frac{C_{m, k}}{t^{(2 k+2 m+1) / 2}} \sum_{n=-\infty}^{\infty}(\theta+2 \pi n)^{2 k} \exp \left(-(\theta+2 \pi n)^{2} / 2 t\right) .
\end{aligned}
$$

where $C_{m, 0}=(2 m) ! / 2^{m} m !(2 \pi)^{1 / 2}$. Thus

(5) $\frac{\partial^{2 m}}{\partial \theta^{2 m}} p(t,(\rho, 0, z),(\rho, \theta, z))=(-1)^{m} \sum_{k=0}^{m}(-1)^{k} C_{m, k} \sum_{n=-\infty}^{\infty} p_{m, n, k}(\rho, \theta, z)$

where $p_{m, n, k}(\rho, z)$ is given by 


$$
E_{\rho, z}\left[\frac{(\theta+2 \pi n)^{2 k}}{R(t)^{(2 m+2 k+1) / 2}} \exp \left(-(\theta+2 \pi n)^{2} / 2 R(t)\right), \rho(t)=\rho, z(t)=z\right]
$$

and

(6) $\frac{\partial^{2 m}}{\partial \theta^{2 m}} q(t,(\rho, 0, z),(\rho, \theta, z))=(-1)^{m} \sum_{k=0}^{m}(-1)^{k} C_{m, k} \sum_{n=-\infty}^{\infty} q_{m, n, k}(\rho, \theta, z)$

where $q_{m, n, k}(\rho, \theta, z)$ is given by

$$
E_{\rho, z}\left[\frac{(\theta+2 \pi n)^{2 k}}{R(t)^{(2 m+2 k+1) / 2}} \exp \left(-(\theta+2 \pi n)^{2} / 2 R(t)\right), \rho(t)=\rho, z(t)=z, \tau>t\right] .
$$

The densities indicated on the right of (5) and (6) not only exist but are bounded, and the series are absolutely convergent. In fact, by Jensen's inequality,

$$
\begin{aligned}
& \left|E_{\rho, z}\left[R(t)^{-\alpha} h(\rho(t), z(t))\right]\right| \\
& \leqq t^{-\alpha-1} E_{\rho, z}\left[\int_{0}^{t} \rho^{2 \alpha}(s) d s|h(\rho(t), z(t))|\right] \\
& =t^{-\alpha-1} \int_{0}^{t} d s E_{\rho, z}\left[\rho^{2 \alpha}(s) E_{\rho(s), z(s)}[|h(\rho(t-s), z(t-s))|]\right] \\
& =t^{-\alpha-1} \int_{0}^{t} d s \int_{0}^{\infty}\left(\rho^{\prime}\right)^{2 \alpha+1} p\left(s, \rho, \rho^{\prime}\right) d \rho^{\prime} \\
& \quad \cdot \int_{0}^{\infty} \rho^{\prime \prime} d \rho^{\prime \prime} \int_{-\infty}^{\infty} d z^{\prime} p\left(t-s, \rho^{\prime}, \rho^{\prime \prime}\right) q\left(t, z-z^{\prime}\right)\left|h\left(\rho^{\prime \prime}, z^{\prime}\right)\right|
\end{aligned}
$$

where

$$
p\left(s, \rho, \rho^{\prime}\right)=\frac{1}{2 \pi s} \int_{0}^{2 \pi} \exp \left(-\left(\rho^{2}+\left(\rho^{\prime}\right)^{2}-2 \rho \rho^{\prime} \cos \theta\right) / 2 s\right) d \theta
$$

and

$$
g(t, z)=(2 \pi t)^{-1 / 2} \exp \left(-z^{2} / 2 t\right) .
$$

Elementary estimation now yields

$$
\begin{aligned}
& \left|E_{\rho, z}\left[(R(t))^{-\alpha} h(\rho(t), z(t))\right]\right| \\
& \quad \leqq A_{\alpha}(t, \rho) \int_{0}^{\infty} \rho^{\prime} d \rho^{\prime} \int_{-\infty}^{\infty} d z^{\prime} p\left(t, \rho, \rho^{\prime}\right) g\left(t, z-z^{\prime}\right)\left|h\left(\rho^{\prime}, z^{\prime}\right)\right|
\end{aligned}
$$

where $A_{\alpha}(t, \rho)$ can be chosen to depend continuously on $(t, \rho) \in(0, \infty)$ $\times(0, \infty)$. This shows that 


$$
E_{\rho, z}\left[R(t)^{-\alpha}, \rho(t)=\rho, z(t)=z\right] \leqq A_{\alpha}(t, \rho) p(t, \rho, \rho) g(t, 0) .
$$

It is obvious from (7) that

(8) $\quad E_{\rho, z}\left[(R(t))^{-\alpha}, \rho(t)=\rho, z(t)=z, \tau>t\right] \leqq A_{\alpha}(t, \rho) p(t, \rho, \rho) g(t, 0)$.

We can now write

$$
\begin{aligned}
& \left.\frac{\partial^{2 m}}{\partial \theta^{2 m}} p(t,(\rho, 0, z),(\rho, \theta, z))\right|_{\theta=0} \\
& \quad=(-1)^{m} C_{m, 0} E_{\rho, z}\left[R(t)^{-(2 m+1) / 2}, \rho(t)=\rho, z(t)=z\right]+\Delta_{m}(t, \rho, z)
\end{aligned}
$$

and

$$
\begin{aligned}
& \left.\frac{\partial^{2 m}}{\partial \theta^{2 m}} q(t,(\rho, 0, z),(\rho, \theta, z))\right|_{\theta=0} \\
& =(-1)^{m} C_{m, 0} E_{\rho, z}\left[R(t)^{-(2 m+1) / 2}, \rho(t)=\rho, z(t)=z, \tau>t\right]+\Delta_{m}^{\prime}(t, \rho, z),
\end{aligned}
$$

where

$$
\Delta_{m}(t, \rho, z)=2(-1)^{m} \sum_{k=0}^{m}(-1)^{k} C_{m, k} \sum_{n=1}^{\infty} p_{m, n, k}(\rho, 0, z)
$$

and

$$
\Delta_{m}^{\prime}(t, \rho, z)=2(-1)^{m} \sum_{k=0}^{m}(-1)^{k} C_{m, k} \sum_{n=1}^{\infty} q_{m, n, k}(\rho, 0, z) .
$$

Since

$$
\sup _{s>0} \frac{(2 \pi)^{2 k}}{s^{(2 m+2 k+1) / 2}} \exp \left(-\left(2 \pi_{n}\right)^{2} / 2 s\right) \leqq B_{m, k} \frac{1}{n^{2 m+1}},
$$

one sees that for $m \geqq 1$ the series defining $\Delta_{m}$ and $\Delta_{m}^{\prime}$ are absolutely convergent, and $\left|\Delta_{m}(t, \rho, z)\right| \vee\left|\Delta_{m}^{\prime}(t, \rho, z)\right| \leqq C_{m} / t$.

In particular, if $m \geqq 1$, then

$$
\begin{aligned}
& \left|\frac{\partial^{2 m}}{\partial \theta^{2 m}} q(t,(\rho, 0, z),(\rho, \theta, z))\right|_{\theta=0} \mid \\
& \quad \leqq C_{m, 0} E_{\rho, z}\left|R(t)^{-(2 m+1) / 2}, \rho(t)=\rho, z(t)=z, \tau>t\right|+\left|\Delta_{m}^{\prime}(t, \rho, z)\right| \\
& \quad \leqq\left|\frac{\partial^{2 m}}{\partial \theta^{2 m}} p(t,(\rho, 0, z),(\rho, \theta, z))\right|_{\theta=0}-\Delta_{m}(t, \rho, z)|+| \Delta_{m}^{\prime}(t, \rho, z) \mid \\
& \quad \leqq\left|\frac{\partial^{2 m}}{\partial \theta^{2 m}} p(t,(\rho, 0, z),(\rho, \theta, z))\right|_{\theta=0} \mid+\frac{C_{m}}{t} .
\end{aligned}
$$

This is the estimate given in (2). 
Derivation of (1). We now want to show that

$$
\lim _{t \sim 0} \frac{\left.\left(\partial^{2 m} / \partial \theta^{2 m}\right) q(t,(\rho, 0, z),(\rho, \theta, z))\right|_{\theta=0}}{\left.\left(\partial^{2 m} / \partial \theta^{2 m}\right) p(t,(\rho, 0, z),(\rho, \theta, z))\right|_{\theta=0}}=1
$$

for $0<z<L$ and $0<\rho<f(z)$. In order to do this, let $P_{\rho, \theta, z}$ be the measure induced on $C\left([0, \infty),(0, \infty) \times S^{\prime} \times R\right)$ by the process $(\rho(t)$, $\theta(R(t)), z(t))$, where $(\rho(t), z(t))$ is governed by $P_{\rho, z}$ and $\theta(t)$ is an independent Brownian motion of $S^{\prime}$ starting at $\theta$. Let $\tau=\inf \{t \geqq 0: \rho(t) \geqq f(z(t))\}$. Then an application to the strong Markov property yields

$$
\begin{aligned}
& q(t,(\rho, 0, z),(\rho, \theta, z)) \\
& =p(t,(\rho, 0, z),(\rho, \theta, z))-E_{\rho, 0, z}\left[\chi_{\tau \leqq t} p(t-\tau,(\rho(\tau), \theta(\tau), z(\tau)),(\rho, \theta, z))\right] .
\end{aligned}
$$

Noting that $\left.\left(\partial^{2 m} / \partial \theta^{2 m}\right) p(t-\tau,(\rho(\tau), \theta(\tau), z(\tau)),(\rho, \theta, z))\right|_{\theta=0}$ contains a factor of the form

$$
\exp \left(-\left(\rho^{2}(\tau)+\rho^{2}-2 \rho(\tau) \rho \cos \theta(\tau)\right) / 2(t-\tau)\right) \leqq \exp \left(-\delta^{2} / 2 t\right),
$$

where $\delta$ is the distance from $(\rho, 0, z)$ to the boundary of $\mathscr{G}$, one can easily see that (11) holds. One might suspect that (12) can also be used to derive (2). However, it is not clear how to get an estimate which is uniform as $(\rho, 0, z)$ tends to the boundary.

To complete the proof of (1), let

$$
q_{m}(t, \rho, z)=\left.\frac{\partial^{2 m}}{\partial \theta^{2 m}} q(t,(\rho, 0, z),(\rho, \theta, z))\right|_{\theta=0}
$$

and

$$
p_{m}(t, \rho, z)=\left.\frac{\partial^{2 m}}{\partial \theta^{2 m}} p(t,(\rho, 0, z),(\rho, \theta, z))\right|_{\theta=0}=\sum_{k=1}^{m} A_{m, k} \rho^{2 k} t^{-(2 k+3) / 2}
$$

and set $U_{\varepsilon}(t)=\left\{(\rho, z): 0<z<L, 0<\rho<f(z)\right.$, and $\left.\left|q_{m} / p_{m}-1\right| \geqq \varepsilon\right\}$. We have just seen that $\left|U_{\varepsilon}(t)\right| \rightarrow 0$ as $\mathrm{t} \rightarrow 0$. Using (2), one has

$$
\begin{aligned}
\left|1-\frac{\int_{0}^{L} d z \int_{0}^{f(z)} q_{m} \rho d \rho}{\int_{0}^{L} d z \int_{0}^{f(z)} p_{m} \rho d \rho \mid}\right| & \leqq \varepsilon \frac{\int_{0}^{L} d z \int_{0}^{f(z)}\left|p_{m}\right| \rho d \rho}{\left|\int_{0}^{L} d z \int_{0}^{f(z)} p_{m} \rho d \rho\right|} \\
+2 \frac{\int_{0}^{L} d z \int_{0}^{f(z)}\left|p_{m}\right| \chi_{U_{\varepsilon}(t)} \rho d \rho}{\left|\int_{0}^{L} d z \int_{0}^{f(z)} p_{m} \rho d \rho\right|}+\frac{C_{m}}{t} & \frac{\left|U_{\varepsilon}(t)\right|}{\left|\int_{0}^{L} d z \int_{0}^{f(z)} p_{m} \rho d \rho\right|} .
\end{aligned}
$$


Multiplying and dividing each of these terms by $t^{m+3 / 2}$ and using the dominated convergence theorem, one sees that they all tend to zero as $t$ and then $\varepsilon$ go to zero.

\section{REFERENCES}

1. M. Kac, Can you hear the shape of a drum?, Amer. Math. Monthly 73 (1966), part II, 1-23. MR 34 \# 1121 .

2. - On applying mathematics: Reflections and examples, Quart. Appl. Math. 30 (1972), 17-29.

3. H. P. McKean, Stochastic integrals, Probability and Math. Statist., no. 5, Academic Press, New York, 1969. MR 40 \#947.

Department of Mathematics, University of Colorado, Boulder, Colorado 80302 